\title{
A non-convex gradient fidelity-based variational model for image contrast enhancement
}

\author{
Qiegen Liu' ${ }^{1}$ Jianbo Liu², Biao Xiong ${ }^{3}$ and Dong Liang ${ }^{2 *}$
}

\begin{abstract}
We propose a novel image contrast enhancement method via non-convex gradient fidelity-based (NGF) variational model which consists of the data fidelity term and the NGF regularization. The NGF prior assumes that the gradient of the desired image is close to the multiplication of the gradient of the original image by a scale factor, which is adaptively proportional to the difference of their gradients. The presented variational model can be viewed as a data-driven alpha-rooting method in the gradient domain. An augmented Lagrangian method is proposed to address this optimization issue by first transforming the unconstrained problem to an equivalent constrained problem and then applying an alternating direction method to iteratively solve the subproblems. Experimental results on a number of images consistently demonstrate that the proposed algorithm can efficiently obtain visual pleasure results and achieve favorable performance than the current state-of-the-art methods.
\end{abstract}

Keywords: Contrast enhancement; Variational model; Augmented Lagrangian method

\section{Introduction}

Image enhancement is an important issue in many fields like computer vision, pattern recognition, and medical image processing. It aims to make the resultant quality better than the original image for a specific application or a set of objectives, where the source of degradation may be unknown. Many images such as medical images, remote sensing images, electron microscopy images, and even real life photographic pictures suffer from poor contrast. Therefore, it is necessary to enhance the contrast to obtain a more visually pleasing image [1].

\subsection{Related work}

Many image contrast enhancement techniques are available which can be roughly be classified into two categories, i.e., spatial-domain algorithm and transformdomain algorithm.

Spatial-domain techniques such as the power law transform and histogram equalization (HE) [1] directly deal with the image pixels by manipulating them to achieve

\footnotetext{
*Correspondence: dong.liang@siat.ac.cn

2 Paul C. Lauterbur Research Center for Biomedical Imaging, SIAT, Chinese Academy of Sciences, Beijing 518055, China

Full list of author information is available at the end of the article
}

the desired enhancement. This category of algorithms is particularly useful for directly altering the gray level values of individual pixels and hence the overall contrast of the entire image. However, they usually enhance the whole image in a uniform manner which may produce undesirable results in many cases. Since then, several improved approaches have been developed [2-5]. For instance, by regarding each sub-histogram as a class, Menotti et al. [3] developed a local-adaptive method that first partitions the overall histogram into multiple sub-histograms by minimizing within-class variance and then applies HE to each sub-histogram separately. As a generalization of $\mathrm{HE}$, the enhancement process of 2DHE is based on the observation that contrast of an image can be improved by increasing the gray-level differences between the pixels of an input image and their neighbors [4]. Huang et al. [5] proposed a hybrid histogram modification method by combining power law transform and HE.

Transform-domain algorithms are related to the data domain they are applied, which may be in frequency domain, discrete cosine transform (DCT) or wavelet transform [6-9]. Alpha-rooting (AR) algorithm is a simple but effective technique for image enhancement in the transform or frequency domain. This algorithm is based

\section{照 Springer}

(c) 2014 Liu et al.: licensee Springer. This is an Open Access article distributed under the terms of the Creative Commons

Attribution License (http://creativecommons.org/licenses/by/4.0), which permits unrestricted use, distribution, and reproduction in any medium, provided the original work is properly credited. 
on the fact that after applying an orthogonal transform to an image, the high frequency coefficients will have smaller magnitudes than the low frequency coefficients; hence, these coefficients may be more amplified to reveal details [6]. Additionally, contrast enhancement algorithms in the DCT domain have attracted many researchers since DCT is adopted in the JPEG compression standard. However, they often introduce unfavorable blocking artifacts, e.g., multi-contrast enhancement method (MCE) $[7,8]$.

Some approaches treat contrast enhancement as an optimization problem. For example, the method of flattest histogram specification with accurate brightness preservation (FHSABP) [10] formulates the transformation of the input image histogram into the flattest histogram as a convex optimization, subject to a mean brightness constraint. Contrast enhancement in histogram modification framework (HMF) is also treated as an optimization problem that minimizes a cost function to address noise and black/white stretching [3]. The contextual and variational contrast $(\mathrm{CVC})$ method enhances the image contrast by constraining a 2D histogram of the input image [11]. A smooth 2D target histogram is obtained by minimizing the sum of the Frobenius norms of the difference from the input histogram and the uniformly distributed histogram. However, it requires a high level of computation when increasing the gray-level differences between neighboring pixels. Unlike the above three methods of formulating optimization with $1 \mathrm{D}$ or $2 \mathrm{D}$ histogram as variables, in this paper, we utilize the image gradient as a optimization variable for contrast enhancement, where the solution is obtained by minimizing the data fidelity as well as the sum of norms of the difference from datadriven weighting-multiplication of the input gradients and the target gradients.

\subsection{Contributions}

In previous works, several researchers suggested applying the gradient fidelity to improve the performance of the algorithm in image denoising and deblurring $[12,13]$. Nevertheless, their improvement is limited. In this work, we refocus on the gradient fidelity and apply it in visual contrast enhancement. The main contributions of this paper are the following:

- For better contrast enhancement, we introduce a novel image prior, the non-convex gradient fidelity (NGF), which assumes that the gradient of the desired image is close to the multiplication of the gradient of the original image by a scale factor, which is adaptively proportional to the difference of their gradients. A data-driven variational model for contrast enhancement is then formulated by combining the data-fidelity term and this prior.
- The difference between the NGF and the conventional AR is revealed. Compared to the AR that is usually operated in Fourier and DCT domain and gives an analytical solution, NGF is data-driven, operated in gradient domain and its optimal solution is achieved by minimizing penalty functions.

- An efficient alternating algorithm is developed to solve the non-linear optimization problem with the advantage of fast convergence. The adaptive model and efficient implementation indicate the potential of the method to be applied in real-time image/video applications.

\section{Non-convex gradient fidelity regularization}

In this section, after briefly surveying some previous results employing gradient fidelity-based prior in image processing, we state our motivation and subsequently propose a NGF variational model for efficient contrast enhancement. Then, the potential relation between NGF and the traditional AR method is explained and an iterative algorithm is developed to efficiently solve it.

\subsection{Proposed model NGF}

Until recently, there were several works utilizing the gradient fidelity-based prior in image processing [12-15]. In [14], Fattal et al. presented a method for rendering high dynamic range (HDR) compression. After manipulating the gradient field of images by attenuating the magnitudes of large gradients, they proposed a gradient fidelity-based functional to reconstruct the result image from the modified gradient information in the least square sense. In [12], Didas et al. combined $\ell_{2}$ data and gradient fitting in conjunction with $\ell_{1}$ regularization for image denoising, where the gradient fidelity term is used to compensate for the loss of edge and undesirable staircase effect introduced by the resulting higher partial differential equation. In [13], $\mathrm{Xu}$ and Jia used one previously predicted sharp edge gradient as a spatial prior to guide the recovery of a coarse version of the latent image for robust motion deblurring. All the methods above could be considered as constructing an image from the specific gradient fields, and their motivations are quite different to ours. The authors in [12] aimed to recover the true image by minimizing gradient fidelity term between the corrupted image and the solution. The authors in [13] and [14] first used some filters to produce a guide image and then applied gradient fidelity term between the guide image and the solution.

Let us turn back to the topic of contrast enhancement. In this work, unlike the previously proposed gradient fidelity term which was measured by linear and convex $\ell_{2}$-norm, we use the non-convex gradient fidelity as a primary regularizer and apply it to enhance contrast. Intuitively, the objective of an enhancement approach is to produce an output image that looks better than the 
original one by increasing the gray-level differences (i.e., the contrast) among pattern objects. To achieve this goal, the relation (or mapping function) between the gradient of the input image and the desired solution should be nonlinear. A simple and straightforward assumption is that the mapping is measured by a weighting function with regard to the difference of their gradients. Mathematically, given the degraded image $f$ and the desired solution $x$, the objective function we used is modeled as follows:

$$
\min _{x}\left\{\|f-x\|_{2}^{2}+\eta \sum_{i=1}^{n^{2}}\left\|\left|D_{i} f-D_{i} x\right|^{\alpha-1} \otimes D_{i} f-D_{i} x\right\|_{2}^{2}\right\}
$$

where $\eta$ is a weighting factor. For each $i, D_{i} x \in R^{2}$ represents the first-order finite difference of $x$ at pixel $i$ in both horizontal and vertical directions. The symbol $\otimes$ denotes point-wise multiplication. The first term in the cost function enforces data fidelity in image-domain. The second term suggests that the target image and the original image should be close with a scale factor which is adaptively proportional to the difference of their gradients. When letting $\alpha=1$, then model (1) degrades to the classical gradient fidelity model as used in $[12,13,15]$. In the circumstance of $\alpha<1$, the weight matrix $w_{i}=\left|D_{i} f-D_{i} x\right|^{\alpha-1}$ measures the distance between the blurred and desired clean image at each pixel $i$.

Interestingly, the NGF regularization in the proposed model (1) essentially differs from the classical AR algorithm [6], which is defined as $\Psi x_{i}=\left|\Psi f_{i}\right|^{\alpha-1} \otimes \Psi f_{i}$, $\alpha>0, \Psi$ is the Fourier or DCT transform. The NGF prior differs from AR algorithm at three aspects: (i) NGF is operated in the gradient domain, while the AR is previously applied in the frequency or DCT domain; (ii) the weight $\left|D_{i} f-D_{i} x\right|^{\alpha-1}$ in NGF is the function not only with respect to the original image but also the desired image which needs to be solved. Particularly, this modification introduces two implicit changes. One change is that $\left|D_{i} f-D_{i} x\right|$ rather than $\left|D_{i} f\right|$ is a relative value, which is more adaptive. The other is that the range of $\alpha$ in the traditional AR method is $\alpha>0$, while being extended to be negative in NGF; and (iii) NGF in model (1) is heavily non-linear and non-convex, and the solution is achieved by modeling it as a variational energy, hence no analytical solution can be directly obtained as that in AR method. In NGF, the term (ii) focuses on the local adaptation and at the same time the term (iii) devotes to the global constraint.

This data-driven based gradient fidelity prior produces enhanced gradients and consequently improves visual contrast. One iteration step of the model (1), which is derived by employing augmented Lagrangian (AL) scheme and alternative direction method in the next subsection, is illustrated in Figure 1. As expected and shown in Figure 1b,c,d, after multiplication with the weight presented in Figure 1c, the gradients in horizontal and vertical directions are more contrast visually near the edge and texture regions. After several iterations, as seen from the original image Figure 1a to the intermediated image Figure 1e obtained by the algorithm, the visual effect is much more improved.

\subsection{Solver}

Although the NGF regularized model has exhibited some appealing properties in model setting, the issues of computational complexity and local optimality have to be addressed since these issues limit its practical application. Therefore, developing an efficient and robust solver is highly desirable. In this subsection, an AL method is proposed to solve the problem. AL method is a well studied optimization algorithm for solving the constrained problems in mathematical programming community [16]. Recently, it is enjoying a re-popularization mainly due to the work of Yin et al. [17] and has been used in various applications of signal/image processing [18,19]. In this work, we use a combination of the reweighted technique and AL scheme, which has been successfully used in [19].

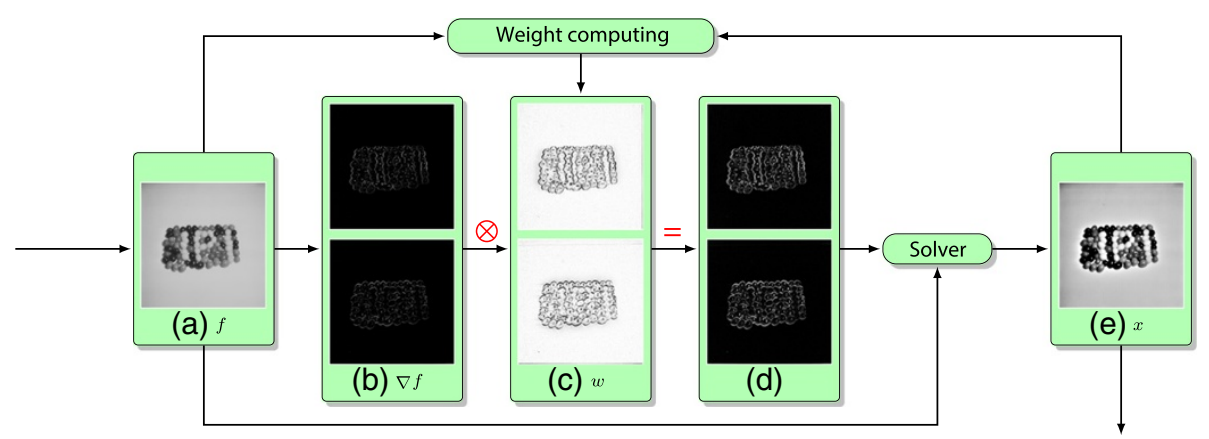

Figure 1 The flow chart of the proposed model. (a) The input/original image $f$. (b) The original gradients $D_{i} f$ in horizontal and vertical directions. (c) The data-driven weight matrix $w_{i}=\left|D_{i} f-D_{i} x\right|^{\alpha-1}$. (d) Enhanced gradients $w_{i} \otimes D_{i} f$. (e) The intermediate image enhanced by solving model (1). 
The AL-related methods usually employ the operator splitting first to transform the original unconstrained minimization problem to an equivalent constrained problem, and then the alternating-minimization strategy is used to iteratively find solutions of the subproblems. Generally speaking, the AL scheme aims to solve the following problem:

$$
\begin{aligned}
& \min _{x, y}\left\{\|f-x\|^{2}+\eta \sum_{i=1}^{n^{2}}\left\|\left|D_{i} f-y_{i}\right|^{\alpha-1} \otimes D_{i} f-y_{i}\right\|^{2}\right\} \\
& \text { s.t. } \quad y_{i}=D_{i} x, i=1, \cdots, n^{2}
\end{aligned}
$$

where $y_{i} \in R^{2}, i=1, \cdots, n^{2}$ are auxiliary variables. Problem (2) can be solved via the standard AL method. Specifically, letting $y=\left[y_{1}, \cdots, y_{n^{2}}\right], \lambda=\left[\lambda_{1}, \cdots, \lambda_{n^{2}}\right]$ and starting from $\lambda^{0}=0$, it solves

$$
\begin{gathered}
\left(x^{k+1}, y^{k+1}\right)=\underset{x, y}{\operatorname{argmin}} L\left(x, y, \lambda^{k}\right)=\underset{x, y}{\operatorname{argmin}}\left\{\|f-x\|^{2}\right. \\
\left.+\sum_{i=1}^{n^{2}}\left(\eta\left\|\left|D_{i} f-y_{i}\right|^{\alpha-1} \otimes D_{i} f-y_{i}\right\|^{2}+\beta\left\|y_{i}-D_{i} x-\lambda_{i}^{k} / \beta\right\|^{2}\right)\right\}
\end{gathered}
$$

at the $k$-th iteration for $\left(x^{k+1}, y^{k+1}\right)$, then updates the multipliers $\lambda$ by the formula

$$
\lambda^{k+1}=\lambda^{k}-\beta\left(y^{k+1}-D x^{k+1}\right)
$$

The sub-problem (3) involves non-convex optimization. In this work, we apply iteratively reweighted method at each AL iteration as used in [19]. Specially at Equation 3, the term $\left|D_{i} f-y_{i}\right|^{\alpha-1}$ is approximated by its latest value
$w_{i}^{k}=\left|D_{i} f-y_{i}^{k}\right|^{\alpha-1}$. Consequently, the AL function (3) is reduced to

$$
\begin{aligned}
\left(x^{k+1}, y^{k+1}\right)= & \underset{x, y}{\operatorname{argmin}}\left\{\|f-x\|_{2}^{2}+\sum_{i=1}^{n^{2}}\left(\eta\left\|w_{i}^{k} \otimes D_{i} f-y_{i}\right\|^{2}\right.\right. \\
& \left.\left.+\beta\left\|y_{i}-D_{i} x-\lambda_{i}^{k} / \beta\right\|^{2}\right)\right\}
\end{aligned}
$$

Since solving (5) for $x$ and $y$ simultaneously can be difficult, an alternative choice is the alternating direction method (ADM) $[18,19]$ that minimizes it with respect to one variable at a time while fixing the other variables at its latest value.

\section{- $x$-subproblem}

By extracting the objective term in Equation 5 with respect to $x$, it yields

$$
\min _{x}\|f-x\|_{2}^{2}+\beta \sum_{i=1}^{n^{2}}\left\|y_{i}-D_{i} x-\lambda^{k} / \beta\right\|^{2}
$$

By taking the derivative of Equation 6 with respect to $x$ and setting it to be zero,

$$
\left(\beta D^{T} D+1\right) x=\beta D^{T}\left(y^{k+1}-\lambda^{k} / \beta\right)+f
$$

We can get the following update rule:

$$
x^{k+1}=\mathcal{F}^{-1}\left(\frac{\mathcal{F}\left(\beta D^{T}\left(y^{k+1}-\lambda^{k} / \beta\right)+f\right)}{\beta \mathcal{F}(D)^{\star} \otimes \mathcal{F}(D)+1}\right)
$$

where $\mathcal{F}$ represents the two-dimensional discrete Fourier transform. The symbol $\star$ denotes complex conjugacy. Both the $\otimes$ and the division signs are component-wise operations.

\section{- $y$-subproblem}

The minimization of Equation 5 with respect to $y$ can be computed analytically. Concretely, we obtain the following optimal solution:

$$
y_{i}^{k+1}=\left(\eta w_{i}^{k} \otimes D_{i} f+\beta D_{i} x^{k}+\lambda^{k}\right) /(\eta+\beta)
$$

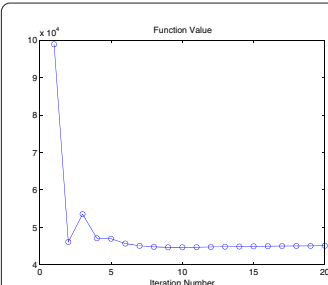

(a)
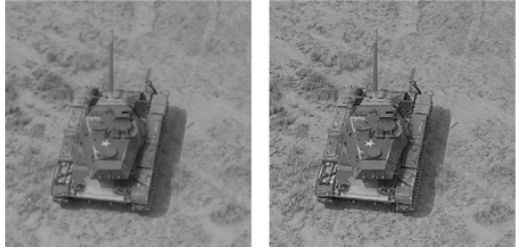

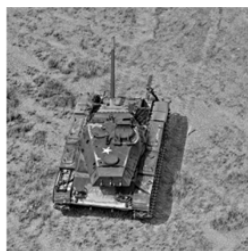

(b)

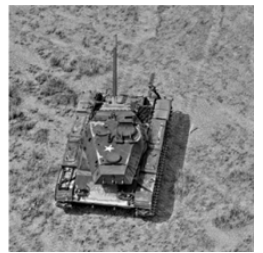

Figure 2 The plot of function values vs iteration number and intermediate enhancement images at different iterations. (a) Objective value evolution. (b) The original image and intimidate images obtained by NGF at first, fourth, 8th, and 16th iterations. 


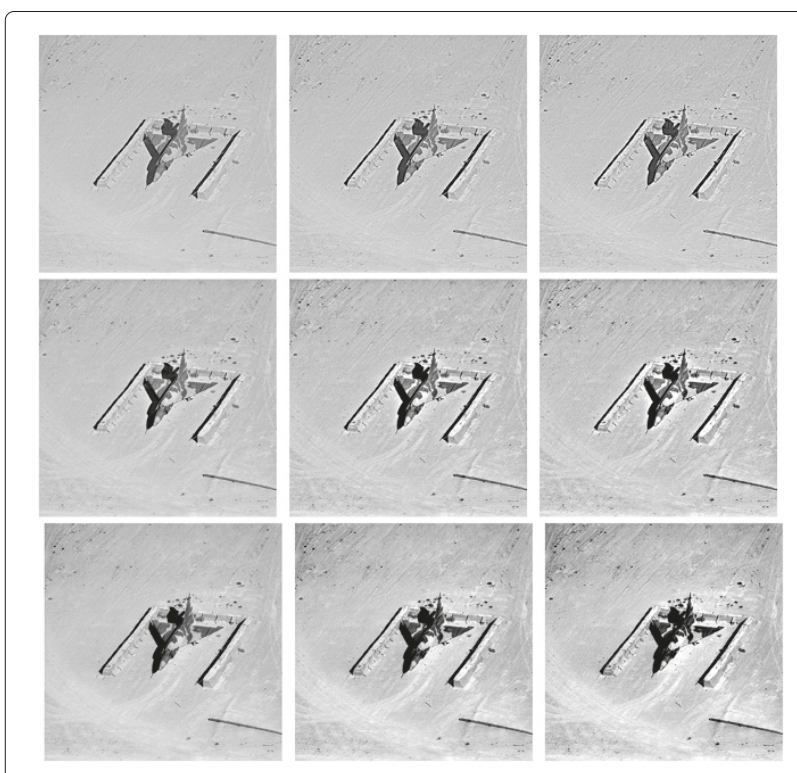

Figure 3 The effect of varying parameters. The results are obtained by varying parameters, where parameter $\eta$ takes 5,100 and 5,000 from top to bottom, and $\alpha$ takes $0.5,0$, and -0.5 from left to right.

In summary, the whole NGF method is summarized as follows:

\begin{tabular}{l}
\hline Algorithm 1 Algorithm NGF \\
\hline 1: for $k=0$ to $K-1$ do do \\
2: update $w_{i}^{k}$ and $y^{k+1}$ according to Equation 9 \\
3: update $x^{k+1}$ according to Equation 8 \\
4: update $\lambda^{k+1}$ according to Equation 4 \\
5: end for \\
\hline
\end{tabular}

\subsection{Computation cost, convergence, and parameter setting}

At each iteration, the computational cost of Equation 9 is linear with respect to problem size, namely $O\left(n^{2}\right)$. Additionally, the main cost for solving Equation 8 is two fast Fourier transforms (FFTs) (including one inverse FFT), and each is at a cost of $O\left(n^{2} \log (n)\right)$, hence the method enables real-time processing. When working on the color images such as in the standard RGB domain, the variable $x \in R^{n^{2}}$ will be extended to $x=\left[x^{r} ; x^{g} ; x^{b}\right] \in R^{3 n^{2}}$. This extension is the same as that in [20].

The convergence property of the algorithm was numerically presented in Figure 2a. It can be observed that the objective function values are almost the same after eight iterations. This may depend on the fact that the ADMbased optimization can decrease the objective function rapidly, as mentioned in many references [18-20]. Figure $2 \mathrm{~b}$ displays the intermediate enhanced images obtained at the 1-th, 4-th, 8-th, and 16-th iteration. We can find that NGF quickly enhances the image in the first few iterations, indicating the effectiveness of the alternating strategy adopted by our method. It only requires a small number of simple arithmetic operations and is thus suitable for real-time applications.

As for the convergence, because of the non-convexity and non-linearity of the problem, the global solution may not be found easily. Nevertheless, since the iterative procedure is updated by AL scheme combined with weighted strategy, local minimum is expected to be attained. Both the value of the objective function and the norm of the reconstruction difference between successive iterations can be chosen as the stopping criterion. Admittedly, providing convergence proof of the proposed algorithm is very difficult. We are still working on the theoretic ground of this deeper issue.

In practice, $w_{i}^{k}=\left|D_{i} f-y_{i}^{k}\right|^{\alpha-1}$ was modified to $w_{i}^{k}=1 /\left[\left|D_{i} f-y_{i}^{k}\right|^{1-\alpha}+\varepsilon\right], 0<\varepsilon<0.5$ to prevent the denominator to be zero. In our work, the algorithm was initialized by letting $w_{i}^{0}=1 / \varepsilon, x^{0}=f, \lambda^{0}=0$. It runs $k$ iterations until the relative tolerance satisfies $\left\|x^{k+1}-x^{k}\right\|_{2} /\left\|x^{k+1}\right\|_{2} \leq \zeta$ with $\zeta=10^{-3}$. The setting of parameter $\beta$ can be referred according to [20]. We empirically choose $\beta=100$ in all the experiments of this article.

\section{Experiments}

In this section, the performance of the proposed method is demonstrated on a variety of images, which show wide variations in terms of average image intensity and contrast. We used a data set comprising standard test images from [21-23] to evaluate and compare the proposed algorithm with $\mathrm{HE}^{\mathrm{a}}$ [1], 2DHE ${ }^{\mathrm{a}}$ [4], $\mathrm{AR}^{\mathrm{b}}$ (alpha rooting in DCT domain [6]), and $\mathrm{MCE}^{\mathrm{b}}$ [7]. The parameter setting of the four algorithms were according to $[4,8]$. When

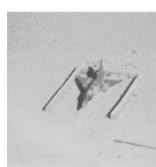

(a)

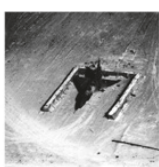

(b)

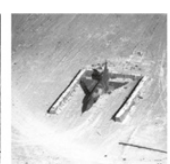

(c)

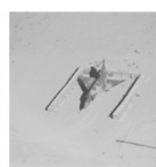

(d)

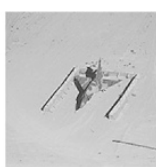

(e)

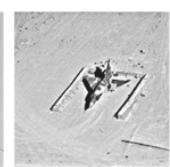

(f)

Figure 4 Contrast enhancement results for image Plane. (a) Original image. (b) HE. (c) $2 D H E$. (d) AR. (e) MCE. (f) NGF. 


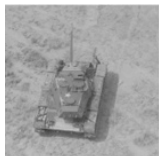

(a)

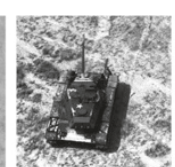

(b)

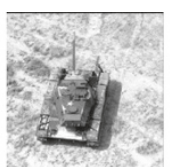

(c)

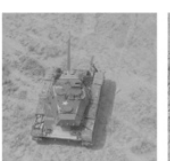

(d)

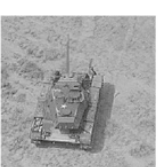

(e)

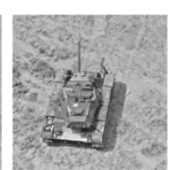

(f)

Figure 5 Contrast enhancement results for image Tank. (a) Original image. (b) HE. (c) 2DHE. (d) AR. (e) MCE. (f) NGF.

extending the gray-level algorithms to color images, $\mathrm{HE}$ and 2DHE first transform the input RGB image to CIE $L^{\star} a^{\star} b^{\star}$ color space, while AR and MCE first transform RGB to be YCbCr space. As analyzed in subsection 2.3, NGF is directly employed in the RGB space.

In the experiments, the performances of these algorithms are investigated in terms of visual quality and quantitative measures. As discussed in a number of papers, the assessment of image enhancement is not an easy task, and there is no any accepted objective criterion that gives meaningful results for every image. Therefore, we choose multiple measures to quantify the improved perception between input image $f$ and output image $x$ as done in [4]. The quantitative measures used in this work include the following: normalized absolute mean brightness error $\operatorname{AMBE}_{N}(f, x)$, normalized discrete entropy $\mathrm{DE}_{\mathrm{N}}$, and normalized edge-based contrast measure $\mathrm{CM}_{\mathrm{N}}(f, x)$. The range of all the three measures is in the interval $[0,1]$. In general, the higher the value of $\mathrm{AMBE}_{\mathrm{N}}$, the better is the brightness preservation, and vice versa. Similarly, higher $D_{E}$ value indicates an estimate image with richer details, and higher $\mathrm{CM}_{\mathrm{N}}$ value indicates an image with higher contrast.

\subsection{Parameter adjustment}

There are two parameters $\eta$ and $\alpha$ in the proposed NGF regularized model. One is used for measuring the level of gradient regularization and the other is for the weighting factor. Therefore, the selection of parameters $(\eta, \alpha)$ consists of a two-dimension parameter space, which

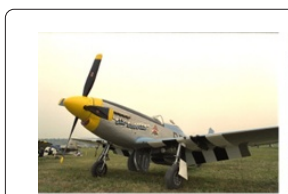

(a)

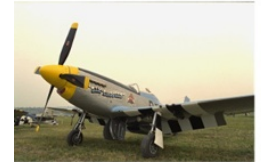

(d)

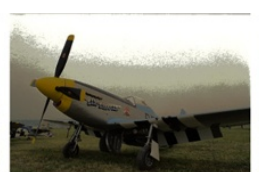

(b)

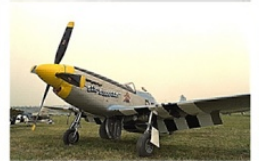

(e)

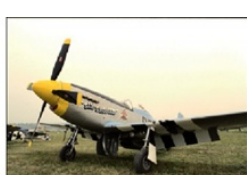

(c)

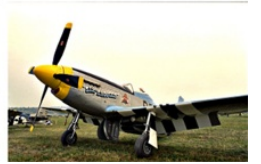

(f)
Figure 6 Contrast enhancement results for image Cessna. (a) Original image. (b) HE. (c) $2 D H E$. (d) AR. (e) MCE. (f) NGF. enables to produce various image style determined by the user.

The effect of varying parameters is demonstrated in Figure 3. The parameter $\eta$ takes 5,100 and 5,000 from top to bottom, and $\alpha$ takes $0.5,0$, and -0.5 from left to right. When $\eta=0$ or $\alpha=1$, the optimal solution of model (1) is the original image itself. We can see that the results with smaller $\eta$ and higher $\alpha$ parameters approximate to the original image (i.e., $\eta \rightarrow 0$ or $\alpha \rightarrow 1$ ). On the other hand, as parameters $\eta$ increasing or/and $\alpha$ decreasing, the contrast in result image is observed to be more and more obvious. In summary, by using different parameters, NGF can achieve different levels of contrast enhancement. In the following experiments, we choose one image with the maximum value according to the discrete entropy and contrast measure (DECM) (i.e., $\operatorname{DECM}_{\mathrm{N}}(f, x)=2 /\left[1 / \mathrm{DE}_{\mathrm{N}}(f, x)+1 / \mathrm{CM}_{\mathrm{N}}(f, x)\right]$ defined in [4]) from the estimates obtained in finite ranges of $(\eta, \alpha)$ as our final result.

\subsection{Comparison on standard test images}

Some contrast enhancement examples resulted from different algorithms for gray-scale images and color images are shown in Figures 4, 5, 6 and 7, respectively.

The original Plane image in Figure 4a shows a lowcontrast image comprising light and dark regions corresponding to ground, plane, and shadow. HE has darkened the image considerably to increase the contrast between regions. Although this method has increased the contrast between different regions of the input image, the

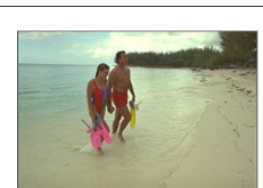

(a)

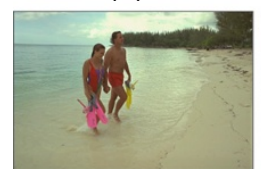

(d)

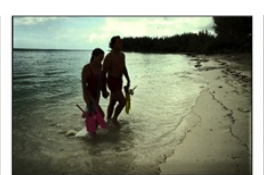

(b)

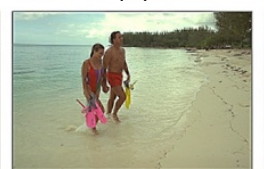

(e)

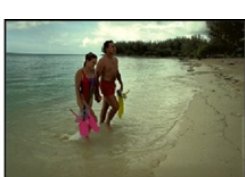

(c)

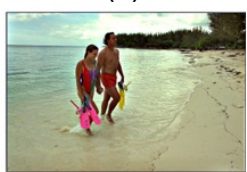

(f)
Figure 7 Contrast enhancement results for image Beach. (a) Original image. (b) HE. (c) $2 \mathrm{DHE}$. (d) AR. (e) MCE. (f) NGF. 
Table 1 Quantitative measurement results of $A M B E_{N}, D E_{N}$, and $C M_{N}$ methods

\begin{tabular}{|c|c|c|c|c|c|c|c|c|c|c|c|c|c|c|c|}
\hline & \multicolumn{5}{|c|}{$\mathrm{AMBE}_{\mathrm{N}}$} & \multicolumn{5}{|c|}{$\mathrm{DE}_{\mathrm{N}}$} & \multicolumn{5}{|c|}{$\mathrm{CM}_{\mathrm{N}}$} \\
\hline & $\mathrm{HE}$ & 2DHE & $A R$ & MCE & NGF & HE & 2DHE & $A R$ & MCE & NGF & $\mathrm{HE}$ & 2DHE & AR & MCE & NGF \\
\hline Plane & 0.0260 & 3269 & 0.5087 & 0.4998 & 0 & 920 & 0.4990 & 5598 & 0.6399 & 0.7693 & 0.5540 & 0.5264 & 0.5010 & 0.5108 & 0.5334 \\
\hline Tank & 0.4928 & 0.0360 & 0.5079 & 0.4998 & 0.6207 & 0.4880 & 0.4950 & 0.5972 & 0.6929 & 0.8120 & 0.5556 & 0.5351 & 0.5017 & 0.5232 & 0.5577 \\
\hline Cam & 0.0944 & 0.0473 & 0.5074 & 0.4957 & 0.4995 & 0.4458 & 0.4812 & 0.4957 & 0.4953 & 0.5490 & 0.5128 & 0.5158 & 0.5015 & 0.5322 & 0.5173 \\
\hline Baboon & 0.3618 & 0.1276 & 0.5079 & 0.4972 & 0.4989 & 0.4572 & 0.4802 & 0.4928 & 0.5058 & 0.7982 & 0.5422 & 0.5266 & 0.5027 & 0.5667 & 0.5362 \\
\hline Cessna & 0.0197 & 0.5973 & 0.5072 & 0.5013 & 0.6877 & 0.4623 & 0.4815 & 0.4916 & 0.4968 & 0.4991 & 0.5220 & 0.5103 & 0.4776 & 0.5060 & 0.5006 \\
\hline Light & 0.1141 & 0.0973 & 0.5052 & 0.5033 & 0.5043 & 0.4502 & 0.4866 & 0.4941 & 0.5554 & 0.5963 & 0.5348 & 0.5192 & 0.5014 & 0.5311 & 0.5365 \\
\hline Beach & 0.0198 & 0.0245 & 0.5064 & 0.5011 & 0.4193 & 0.4528 & 0.4767 & 0.4937 & 0.5571 & 0.6130 & 0.5305 & 0.5164 & 0.5009 & 0.5135 & 0.5427 \\
\hline Island & 0.2495 & 0.1873 & 0.5048 & 0.5016 & 0.5056 & 0.4532 & 0.4670 & 0.4897 & 0.6152 & 0.6748 & 0.5256 & 0.5234 & 0.5015 & 0.5257 & 0.5449 \\
\hline Iverage & 0.1723 & 0.1805 & 0.5069 & 0.5000 & 0.5463 & 0.4627 & 0.4830 & 0.5143 & 0.5698 & 0.6640 & 0.5347 & 0.5217 & 0.4985 & 0.5262 & 0.5337 \\
\hline
\end{tabular}

The best among each results are in italics.

contrast within each region of the image is considerably reduced. For example, the texture on the plane is not identifiable. 2DHE produces a brighter image which has better visual quality and contrast than the result of HE. However, the ahead ground results in a slightly brighter output image. Since AR and MCE are conducted in small DCT blocks, their results do not change the overall contrast of the image well, although it can be observed that some details on the plane are enhanced in the MCE result. Our method improves the overall contrast while preserving the image details. In Figure 4e, it is easy to identify the ground texture as well as the plane.

Figure 5 displays the results of the Tank image. In the HE result shown in Figure 5a, the contrast between the tank and its surrounding is significantly increased. However, the details in the darker area of the tank body are barely noticeable. 2DHE alleviates the drawback of HE by considering the contextual information in the image when producing the $2 \mathrm{D}$ histogram, which makes the details of the tank body better perceived. However, it produces a higher contrast image but brighter image overall, especially on the ground. AR leads to an image that is almost similar to its original, and hence contrast has been poorly improved. MCE retains more detail than the image obtained with AR. However, the photometric difference between the tank and its surrounding is still limited. The output of NGF is visually pleasing and the contrast between the tank and its surrounding is high enough to reveal details on both areas.

The image Cessna in Figure 6a shows a plane on a grass field against a background of sky. The image consists of bright (i.e., the sky) and dark (i.e., shadow and grass) regions. Therefore, it is difficult to discriminate the details on the plane and its surrounding. HE generates an output image with high image degradation, where the sky region with original orange tint has been changed to noticeable layers of colored regions ranging from dark orange to light gray. HE also darkens the input image, making its details difficult to be observed. 2DHE generates an improved output image in the area below the plane with no degradations in the sky. NGF also provides an output image with no image degradation in the sky, and furthermore, the details on the plane are better visible. In particular, the shadow below the plane in our result is much smaller than that in the result of 2DHE. Besides, many block effect-like artifacts are observed in the MCE result.

For the input Beach image as shown in Figure 7a, a darkening effect on the couple and the distant hill occurred in the results of the HE and 2DHE methods, which make details not identifiable. MCE and our proposed NGF considerably increase the overall contrast by making the colors in the image richer and enabling image details to be identified.

The computed quantitative measures $\mathrm{AMBE}_{\mathrm{N}}, \mathrm{DE}_{\mathrm{N}}$, and $\mathrm{CM}_{\mathrm{N}}$ are showed in Table 1. The average $\mathrm{AMBE}_{\mathrm{N}}$ values show that NGF outperforms all other algorithms in brightness preservation. The $\mathrm{DE}_{\mathrm{N}}$ values show that the proposed algorithm outperforms all the other algorithms. In particular, $\mathrm{HE}$ provides the lowest $\mathrm{DE}_{\mathrm{N}}$ value because it groups bins and thus reduces the overall entropy of the output image. The high average value of $\mathrm{DE}_{\mathrm{N}}$ and $\mathrm{AMBE}_{\mathrm{N}}$ shows that the proposed NGF is successful in preserving the contextual information while improving the visual quality. It is worth noting that although HE

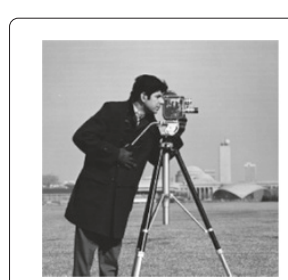

(a)

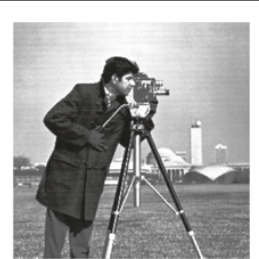

(b)

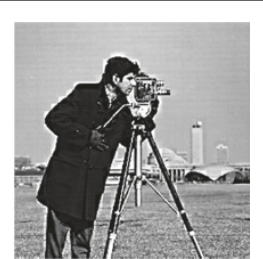

(c)
Figure 8 Contrast enhancement results for image Camera man. (a) Original. (b) HE. (c) MCE. 
Table 2 Average quantitative measurement results on $\mathbf{3 0 0}$ test images from BSDS dataset [23]

\begin{tabular}{cccc}
\hline Method & $\mathbf{A M B E}_{\mathbf{N}}$ & $\mathbf{D E}_{\mathbf{N}}$ & $\mathbf{C M}_{\mathbf{N}}$ \\
\hline $\mathrm{HE}$ & 0.1034 & 0.4496 & 0.5253 \\
$2 \mathrm{DHE}$ & 0.2052 & 0.4822 & 0.5263 \\
$\mathrm{AR}$ & 0.4986 & 0.4296 & 0.5290 \\
$\mathrm{MCE}$ & 0.4982 & 0.4624 & 0.5636 \\
NGF & 0.5051 & 0.6862 & 0.5405 \\
\hline
\end{tabular}

The best among each results are in italics.

and MCE attain high $\mathrm{CM}_{\mathrm{N}}$ values, it does not necessarily mean that they produce visually pleasuring images [4]. Figure 8 shows one example, where HE makes the sky over-whitened and MCE introduces much blocking artifacts near the arm. In order to be convenient for the readers to better judge the effect of these algorithms visually, more supporting numerical illustrations are provided in Additional file 1.

\subsection{Comparison on image database}

In order to evaluate the performance of the five algorithms for a wide range of images, they were applied to 300 test images from Berkeley images dataset [23]. The average measurement values of $\mathrm{AMBE}_{\mathrm{N}}, \mathrm{DE}_{\mathrm{N}}$, and $\mathrm{CM}_{\mathrm{N}}$ are reported in Table 2. Similar to the presented results in Table 1, NGF outperforms the other algorithms in terms of the average $A M B E_{N}$ and $D_{N}$ values. Meanwhile, NGF achieves the second in terms of average $\mathrm{CM}_{\mathrm{N}}$ value. It not only protects the content of the image better than the other algorithms but also exhibits good brightness preservation. More visual comparison examples of these algorithms are included in the Additional file 1.

\subsection{Extension by combining detail enhancement}

As we know, classical unsharp masking techniques aiming at enhancing sharpness/detail of the image usually suffer from the halo effect. Recently, a number of edgepreserving filters like weighted least squares (WLS) [24] have been proposed to alleviate this drawback and achieved impressive performances. As discussed in [25], enhancement of the overall contrast and sharpness of the image are two related but different tasks. On one hand, contrast enhancement does not necessarily lead to sharpness enhancement. On the other hand, when enhancing the sharpness of an image, the noise is also enhanced as well. Hence, combined methods can be attained by integrating NGF and WLS into a unified framework. One possible choice is that after decomposing an image by WLS, we use NGF to level the contrast of its base layer and then combine the remaining detail layers with boosted coefficients (denoted as combined 1). Another choice is to employ NGF first and then use WLS to tackle the intermediate image (denoted as combined 2). These combined strategies may improve the visual quality.

Figure 9 shows one example for demonstrating the superior performance of the combined strategies. Our NGF enhances the contrast locally and globally. WLS attains comparable result with better detail enhancement but introducing little noise. The results from combined methods shown in Figure 9d,e gain better visually pleasing effects. More numerical illustrations are provided in the Additional file 1.

\section{Conclusions}

This work presents the non-convex gradient fidelity term as a regularizer for contrast enhancement. Following the straightforward model and simple implementation, the experimental results obtained for various types of images are highly encouraging and illustrate that our method is superior to the state-of-the-art enhancement techniques. The approach is found to be computationally efficient in producing visually pleasing images.

Since our model is in a variational formulation, further extension is to extend our proposed model by incorporating other penalty priority for some specific applications. For example, by combining the $\ell_{0}$ gradient minimization prior [26], the compound model has the potential to simultaneously enhancing and denoising. Furthermore, it is very desirable to integrate the proposed NGF prior with the recently popular dictionary learning-based sparse representation model $[27,28]$ for texture-enhanced image denoising, as demonstrated in

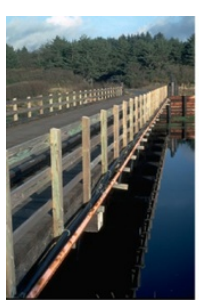

(a)

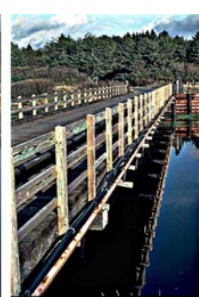

(b)

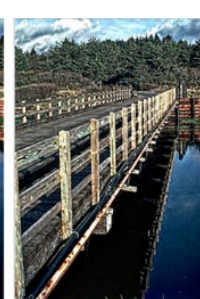

(c)

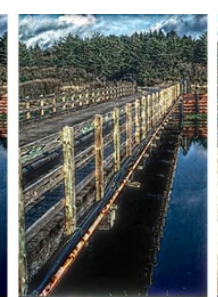

(d)

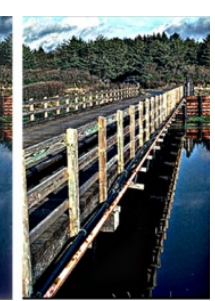

(e)

Figure 9 Contrast or/and sharpness enhancement results for image 22013.jpg. (a) Original. (b) NGF. (c) WLS. (d) Combined 1. (e) Combined 2. 
[27] that a gradient histogram preservation algorithm was presented to enhance the texture structures while removing noise. Applying NGF prior in the trained filters [29] or learned transforms [30] will also be considered in the further study.

\section{Endnotes}

${ }^{\mathrm{a}}$ The codes of HE and 2DHE are available at http:// www.sciencedirect.com/science/article/pii/ S0031320312001525.

b The codes of AR and MCE are available at http:// www.facweb.iitkgp.ernet.in/ jay/CES/.

\section{Additional file}

Additional file 1: Comparison with state-of-the-art algorithms and the extension. The supplementary material contains three parts. Pages 2 to 24 consist of comparison on test images from [1] and [2]. Pages 25 to 50 consist of comparison on test images from [3]. Finally, pages 51 to 55 involves the extension of our method by combining detail enhancement and the comparison with WLS [4] and EAW [5].

\section{Competing interests}

The authors declare that they have no competing interests.

\section{Acknowledgements}

This work was partly supported by the National Natural Science Foundation of China under grant numbers 61261010, 61362001, 61365013, 61340025, and 51165033 , the Natural Science Foundation of Jiangxi province (20132BAB211030, 20121BBE50023, 20122BAB211015), the international scientific and technological cooperation projects of Jiangxi Province (No. 20141BDH80001), the Technology Foundation of the Department of Education in Jiangxi Province (Nos. GJJ13061, GJJ13376, GJJ14196), and the Young Scientist Training Program of Jiangxi province (No.20142BCB23001). The authors are indebted to two anonymous referees for their useful suggestions and for having drawn the authors' attention to additional relevant references.

\section{Author details \\ ${ }^{1}$ Department of Electronic Information Engineering, Nanchang University, Nanchang 330031, China. ${ }^{2}$ Paul C. Lauterbur Research Center for Biomedical Imaging, SIAT, Chinese Academy of Sciences, Beijing 518055, China. ${ }^{3}$ Faculty of Geo-Information Science and Earth Observation-ITC, University of Twente, Enschede, The Netherlands.}

\section{Received: 3 July 2014 Accepted: 4 September 2014}

Published: 10 October 2014

\section{References}

1. RC Gonzalez, RE Woods, Digital Image Processing (3rd Edition). (Prentice-Hall, Inc., Upper Saddle River, 2006)

2. SS Agaian, B Silver, KA Panetta, Transform coefficient histogram-based image enhancement algorithms using contrast entropy. IEEE Trans. Image Process. 16(3), 741-758 (2007)

3. D Menotti, L Najman, J Facon, A De Araujo, Multi-histogram equalization methods for contrast enhancement and brightness preserving. IEEE Trans. Consum. Electron. 53(3), 1186-1194 (2007)

4. TCelik, Two-dimensional histogram equalization and contrast enhancement. Pattern Recognit. 45(10), 3810-3824 (2012)

5. S Huang, F Cheng, Y Chiu, Efficient contrast enhancement using adaptive gamma correction with weighting distribution. IEEE Trans. Image Process. 22(3), 1032-1041 (2013)

6. S Aghagolzadeh, OK Ersoy, Transform image enhancement. Opt. Eng. 31(3), 614-626 (1992)

7. J Tang, E Peli, S Acton, Image enhancement using a contrast measure in the compressed domain. IEEE Signal Process. Lett. 10(10), 289-292 (2003)
8. J Mukherjee, SK Mitra, Enhancement of color images by scaling the DCT coefficients. IEEE Trans. Image Process. 17(10), 1783-1794 (2008)

9. R Fattal, Edge-avoiding wavelets and their applications. ACM Trans. Graph. (TOG) 28(3), 1-10 (2009)

10. CWang, J Peng, Z Ye, Flattest histogram specification with accurate brightness preservation. IET Image Process. 2(5), 249-262 (2008)

11. TCelik, T Tjahjadi, Contextual and variational contrast enhancement. IEEE Trans. Image Process. 20(12), 3431-3441 (2011)

12. S Didas, $S$ Setzer, $G$ Steidl, Combined $\ell_{2}$ data and gradient fitting in conjunction with $\ell_{1}$ regularization. Adv. Comput. Math. 30(1), 79-99 (2009)

13. $L X u, J$ Jia. Two-phase kernel estimation for robust motion deblurring, in Proceedings of European Conference on Computer Vision, (2010), pp. 157-170

14. R Fattal, D Lischinski, $M$ Werman, Gradient domain high dynamic range compression. ACM Trans. Graph. (TOG) 21(3), 249-256 (2002)

15. P Bhat, CL Zitnick, M Cohen, B Curless, Gradientshop: a gradient-domain optimization framework for image and video filtering. ACM Trans. Graph. (TOG) 29(2), 1-14 (2010)

16. RT Rockafellar, Augmented lagrangians and applications of the proximal point algorithm in convex programming. Math. Oper. Res. 1(2), 97-116 (1976)

17. W Yin, S Osher, D Goldfarb, J Darbon, Bregman iterative algorithms for $\ell_{1}$-minimization with applications to compressed sensing. SIAM J. Imaging Sci. 1(1), 143-168 (2008)

18. MV Afonso, JM Bioucas-Dias, MA Figueiredo, An augmented lagrangian approach to the constrained optimization formulation of imaging inverse problems. IEEE Trans. Image Process. 20(3), 681-695 (2011)

19. Q Liu, S Wang, J Luo, Y Zhu, M Ye, An augmented lagrangian approach to general dictionary learning for image denoising. J. Vis. Commun. Image Representation. 23(5), 753-766 (2012)

20. M Tao, J Yang, Alternating direction algorithms for total variation deconvolution in image deconstruction. Optimization Online (2009)

21. The USC-SIPI Image Database. http://sipi.usc.edu/database/

22. Kodak Lossless True Color Image Suite. http://rOk.us/graphics/kodak/

23. D Martin, C Fowlkes, D Tal, J Malik. A database of human segmented natural images and its application to evaluating segmentation algorithms and measuring ecological statistics, in Proceedings of IEEE International Conference on Computer Vision, vol. 2, (2001), pp. 416-423

24. Z Farbman, R Fattal, D Lischinski, R Szeliski, Edge-preserving decompositions for multi-scale tone and detail manipulation. ACM Trans. Graph. (TOG) 27(3), 67-77 (2008)

25. G Deng, A generalized unsharp masking algorithm. IEEE Trans. Image Process. 20(5), 1249-1261 (2011)

26. $L X u, C L u, Y X u, J$ Jia, Image smoothing via $\ell_{0}$ gradient minimization. ACM Trans. Graph. (TOG) 30(6), 174-185 (2011)

27. W Zuo, L Zhang, C Song, D Zhang. Texture enhanced image denoising via gradient histogram preservation, in Proceedings of IEEE Conference on Computer Vision and Pattern Recognition, (2013), pp. 1203-1210

28. R Yan, L Shao, Y Liu, Nonlocal hierachical dictionary learning using wavelets for image denoising. IEEE Trans. Image Process. 22(12), 4689-4698 (2013)

29. L Shao, H Zhang, G De Haan, An overview and performance evaluation of classification-based least squares trained filters. IEEE Trans. Image Process. 17(10), 1772-1782 (2008)

30. L Shao, R Yan, X Li, Y Liu, From heuristic optimization to dictionary learning: a review and comprehensive comparison of image denoising algorithms. IEEE Trans. Cybern. 44(7), 1001-1013 (2014)

\section{doi:10.1186/1687-6180-2014-154}

Cite this article as: Liu et al:: A non-convex gradient fidelity-based variational model for image contrast enhancement. EURASIP Journal on Advances in Signal Processing 2014 2014:154. 